\title{
Oncolytic poxvirus homes in on cancer cells
}

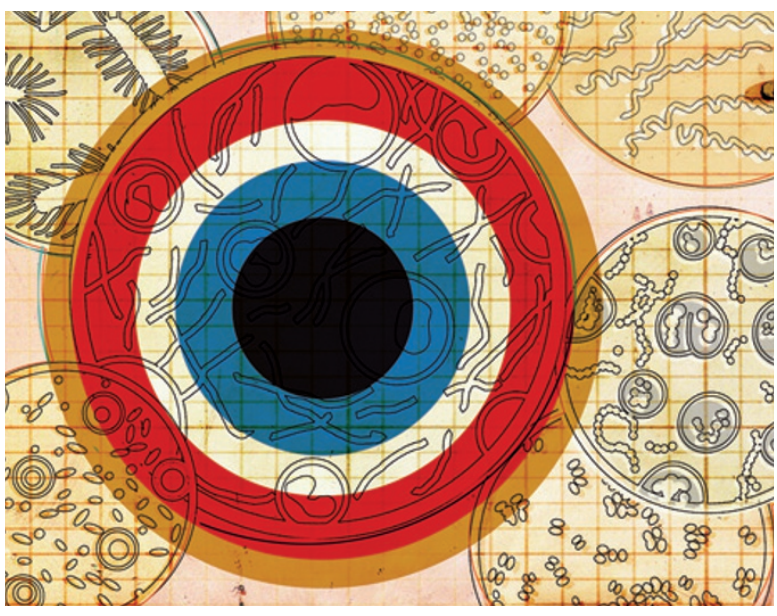

One of the biggest challenges for systemic anticancer treatment is to achieve sufficiently high local concentrations of the drug in the tumours, while sparing healthy tissues. Reporting in Nature, Breitbach et al. demonstrate how this problem can be addressed with a tumourhoming poxvirus engineered to express therapeutic proteins.

The JX-594 virus used in the study is an attenuated version of a live vaccinia virus vaccine that had been used during the smallpox eradication campaign. The vaccinia virus is ideally suited for intravenous (i.v.) delivery as it is resistant to complement and antibody-mediated neutralization in the blood. It can spread to distant tissues, which is important for the treatment of systemic disease, and because of its relatively large size, it is preferentially deposited in tumours, where the neovasculature has increased permeability. Attenuation of JX-594 was achieved through inactivation of its thymidine kinase gene. It was further engineered to express $\beta$-galactosidase, allowing the detection of viral replication, as well as granulocyte-macrophage colony-stimulating factor (GM-CSF) to stimulate antitumour immunity. The selective replication of JX-594 in cancer cells is facilitated by its dependence on the EGFR-RAS pathway, which is commonly activated in epithelial tumours, as well as by thymidine kinase elevation and downregulation of type 1 interferon signalling in many cancer cells.

The virus had previously been tested in a Phase I trial of intratumoural injection in patients with liver cancer, where it was associated with replication in cancer cells, expression of biologically active GM-CSF and tumour destruction. Now, in a Phase I dose-escalation trial of a single i.v. infusion in 23 patients with advanced, treatmentrefractory solid tumours, the authors set out to test whether the virus can be administered in a systemic fashion for the treatment of metastatic disease.

In eight patients who had received the highest doses, delivery and replication of the virus in tumour tissues were confirmed by immunohistochemical staining for $\beta$-galactosidase and by PCR in tumour biopsy samples obtained 8-10 days after treatment. In these patients, tumouradjacent and intermixed normal tissues were negative for viral replication, and immediately adjacent epithelium showed uptake of the virus without replication. Three of these patients had significantly elevated blood levels of GM-CSF. Overall, the authors report dose-related antitumour activity with a reduction in new tumour outgrowth and suppression of microscopic tumour foci in high-dose patients. The treatment was generally well tolerated, with side effects including flu-like symptoms lasting up to 24 hours. Repeated i.v. dosing is currently being assessed.

This study is the first to demonstrate dose-related delivery, replication and transgene expression from a viral vector in metastatic solid tumours in humans after i.v. administration. The authors point out that oncolytic poxviruses can be engineered to express several proteins and/or small interfering RNA, thereby providing a technology platform for the selective expression of high concentrations of complementary therapeutic and imaging molecules in metastatic solid tumours.

Alexandra Flemming

ORIGINAL RESEARCH PAPER Breitbach, C. J. et al. Intravenous delivery of a multi-mechanistic cancer-targeted oncolytic poxvirus in humans. Nature 477, 99-102 (2011)

FURTHER READING Park, B. H. et al. Use of a targeted oncolytic poxvirus, JX-594, in patients with refractory primary or metastatic liver cancer: a phase I trial. Lancet Oncol. 9, 533-542 (2008) 